\title{
Frustrated magnets with chemically tailorable interactions
}

\author{
Alasdair J. Bradford ${ }^{1,2}$, Wenjiao Yao ${ }^{1}$, Ivan da Silva ${ }^{3}$, Mark, Telling ${ }^{4}$, Tatiana Guidi ${ }^{4}$, Duc $\mathrm{Li}^{4}$, Stephen L. Lee ${ }^{2}$ and \\ Philip Lightfoot ${ }^{1}$
}

\author{
${ }^{1}$ School of Chemistry, University of St Andrews, St Andrews, Fife, Scotland, UK, \\ ${ }^{2}$ School of Physics and Astronomy, University of St Andrews, St Andrews, Fife, Scotland, UK, \\ ${ }^{3}$ ISIS Crystallography Group, ISIS Neutron and Muon Source, STFC RAL, Didcot, UK, \\ ${ }^{4}$ ISIS Excitations Group, ISIS Neutron and Muon Source, STFC RAL, Didcot, UK, ajb35@st-andrews.ac.uk
}

In pursuit of new materials in the topical area of low dimensional and frustrated magnetic systems we have begun to investigate a family of materials which use the oxalate ligand as a backbone from which multiple structures can be derived through combination with magnetic spin sources and additional ligand groups. We currently focus primarily on compounds which have first row transition metals as sources of magnetic spin [1][2], which can then be coupled in two-dimensional (2d) layered lattices.

Two such examples we have synthesised are the isostructural compounds $\operatorname{CsM}^{\mathrm{II}}\left(\mathrm{C}_{2} \mathrm{O}_{4}\right) \mathrm{F}(\mathrm{M}=\mathrm{Co}$ or $\mathrm{Fe})$, shown in Fig. 1. These compounds comprise a layered $2 \mathrm{~d}$ structure where magnetic exchange coupling is suppressed between layers by the presence of large $\mathrm{Cs}^{+}$ions. Within the layers, the metal atoms are arranged in a rectangular lattice with the two coupling groups segregated in opposite axial directions (along the $a$ axis $-\mathrm{F}^{-}$coupling, along $b$ axis $-\mathrm{C}_{2} \mathrm{O}_{4}{ }^{2-}$, oxalate coupling). The strong two dimensionality plus the very clean in-plane rectangular coupling means this system in principle maps very nicely onto a $2 \mathrm{~d}$ Heisenberg (3D) $\mathrm{J}_{1} / \mathrm{J}_{1}$ ' type of model, which should be completely free of any diagonal $\left(J_{2}\right)$ exchange coupling that might geometrically frustrate the system [3]. Theoretically this should either lead to an antiferromagnetic stripe order, or with sufficient inter-chain coupling to Néel long-range order (LRO) in the plane, with possible transitions between these states with temperature [3]. Additional coupling within the layers or between the layers may also lead to frustrated ground states, and there have been several theoretical and numerical studies of these kinds of idealised systems [4].
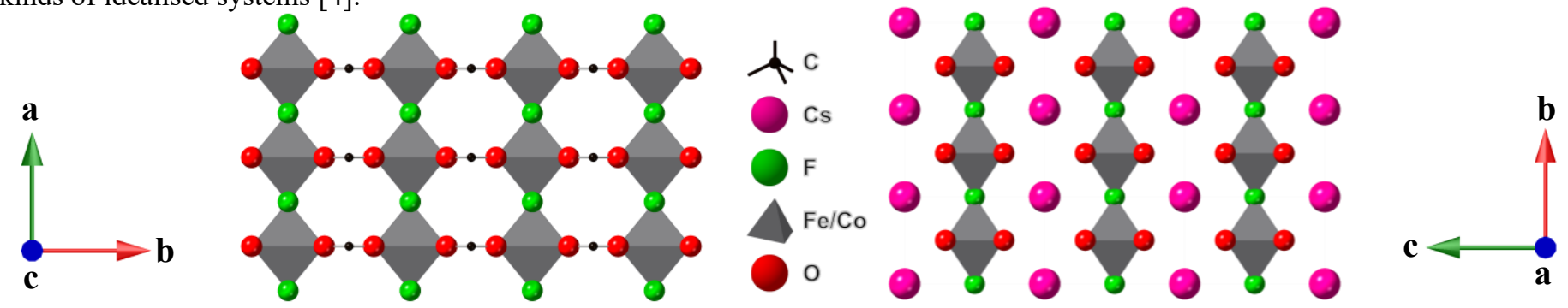

Figure 1. $\mathrm{Cs}[\mathrm{Fe}, \mathrm{Co}]\left(\mathrm{C}_{2} \mathrm{O}_{4}\right) \mathrm{F}$ along $\mathrm{c}$ axis [Left] and along $\mathrm{b}$ axis [Right], [Fe,Co]-[Fe,Co] distances $[4.022,4.035] \AA$ along a, $[5.515,5.407] \AA$ along $\mathrm{b}$ and $[6.467,6.461] \AA$ along $\mathrm{c}$ \{space group Pmmm\}

From elastic neutron powder diffraction data, while the two materials have a near identical nuclear structure, their magnetic superstructures are quite different when measured below their transition temperatures (100-150 K). In the Fe compound, the moments are aligned such that the antiferromagnetic coupling by the $\mathrm{F}^{-}$and $\mathrm{C}_{2} \mathrm{O}_{4}{ }^{2-}$ groups dominates, creating a Néel state square lattice with ferromagnetic alignment between layers. The Co material while maintaining the $\mathrm{F}^{-}$antiferromagnetic coupling, conversely displays ferromagnetic coupling across the $\mathrm{C}_{2} \mathrm{O}_{4}{ }^{2-}$ group. This leads to antiferromagnetic coupling across the $\mathrm{Cs}^{+}$layer. This difference in magnetic structure is currently being investigated by examining system excitations to determine the energy differences between these two states but it is known that the oxalate ligand can support both types of coupling depending on its environment [5][6]. Examining this system more closely may give insight into the mechanisms and limits which govern how systems of this type order generally and so act as a basis for explaining more complex systems which may have frustrated character.

[1] W. Yao et al., Chem. Mater., 2017, 29, 6616.

[2] K. Sustain et al., Inorg. Chem., 2019, 58, 11971.

[3] P. Sindzingre, Phys. Rev. B, 2004, 69, 094418

[4] A. Orendacova et al, Crystals, 2019, 9, 6

[5] M. Peric et al, Monatsh Chem, 2012, 143, 569-577

[6] J. Vallejo et al, Dalton T., 2010, 39, 2350-2358

\section{Keywords: 2D Materials; frustrated magnetism; neutron diffraction}

Thank you to ISIS Neutron and Muon Source and the University of St Andrews for their support of this work

Acta Cryst. (2021), A77, C966 